\title{
Osteomyelitis in elderly patients
}

\author{
C L Romanò , N Logoluso, A Elia, D Romanò \\ From de Senectute: Age and Health Forum \\ Catanzaro, Italy. 5-7 December 2009
}

Older adults are predisposed to osteomyelitis either because of an increased incidence of associated disorders (e.g., peripheral vascular disease, diabetes mellitus, malnutrition and poor dentition) or because of surgical procedures that are frequently performed in the elderly population (e.g., dental extractions, open-heart surgery, and prosthetic joint replacement or osteosynthesis).

Two types of haematogenous osteomyelitis that are seen in the elderly are vertebral and long bone osteomyelitis. The most common pathogen is Staphylococcus aureus.

Osteomyelitis secondary to contiguous foci of infection can occur in older adults without vascular insufficiency (secondary to pressure ulcers) or with vascular insufficiency due to diabetes mellitus or peripheral vascular disease from atherosclerosis.

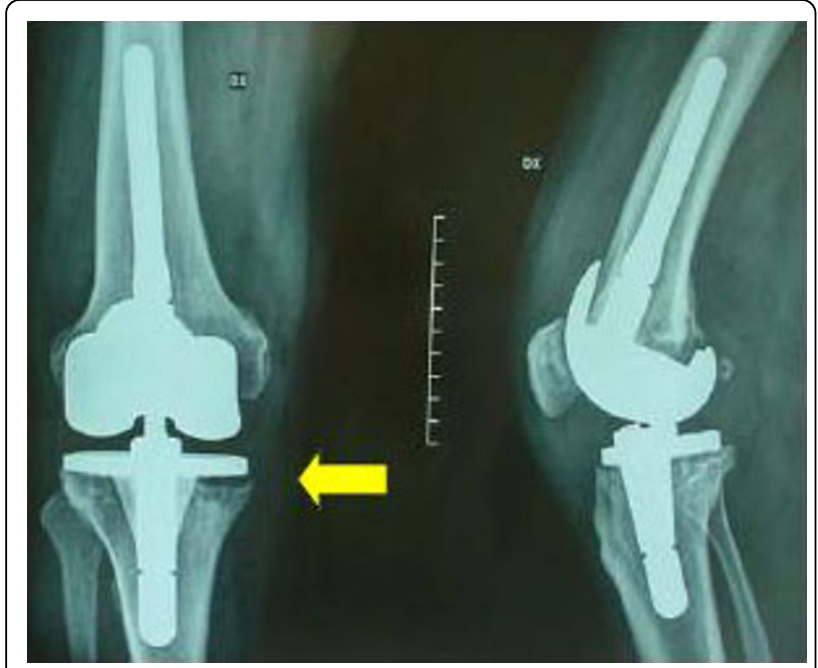

Figure 1 Septic knee prosthesis. The arrow shows the bone loss, due to infection and loosening of the implant.

Centro di Chirurgia Ricostruttiva e delle Infezioni Osteo-articolari, Istituto Ortopedico IRCCS Galeazzi, 20166-Milano, Italy
Chronic osteomyelitis may be defined as osteomyelitis that has a duration of more than 6 weeks or that recurs or is not cured after the initial infection. Chronic osteomyelitis may be associated with certain surgical procedures (e.g., sternal osteomyelitis after open-heart surgery or implant-related infections after orthopaedic and traumatological procedures), poor dentition or dental extraction (mandibular osteomyelitis), and, more commonly, with systemic disorders (e.g., peripheral vascular disease and diabetes mellitus).

Acute osteomyelitis can in some cases be cured with antimicrobial therapy alone but surgical management is often required and includes extensive debridement, obliteration of dead spaces, stabilisation, adequate soft tissue coverage, and restoration of an effective blood supply.

Chronic osteomyelitis may be caused by S. aureus or coagulase negative staphylococci, but is often due to gram-negative organisms. Because of the presence of infected bone fragments without a blood supply (sequestra), cure with antibiotic therapy alone is rare, if ever, possible and adequate surgical debridement is the cornerstone of therapy.

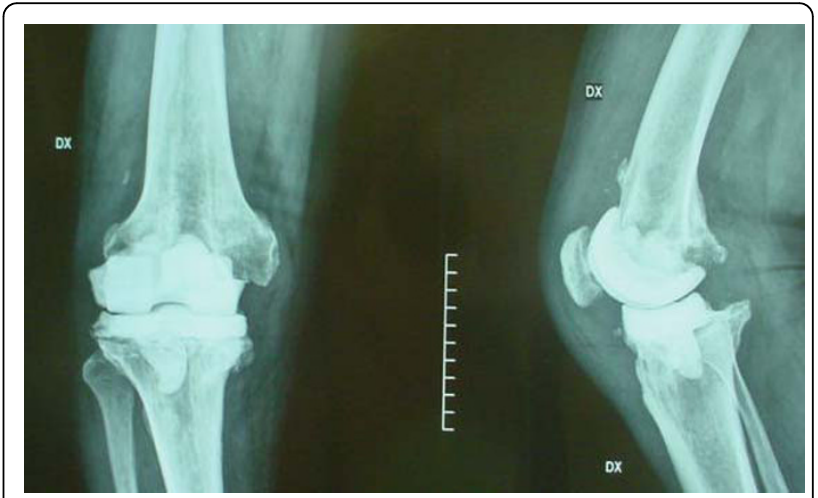

Figure $\mathbf{2}$ X-ray after septic prosthesis removal and antibiotic-loaded spacer implant 


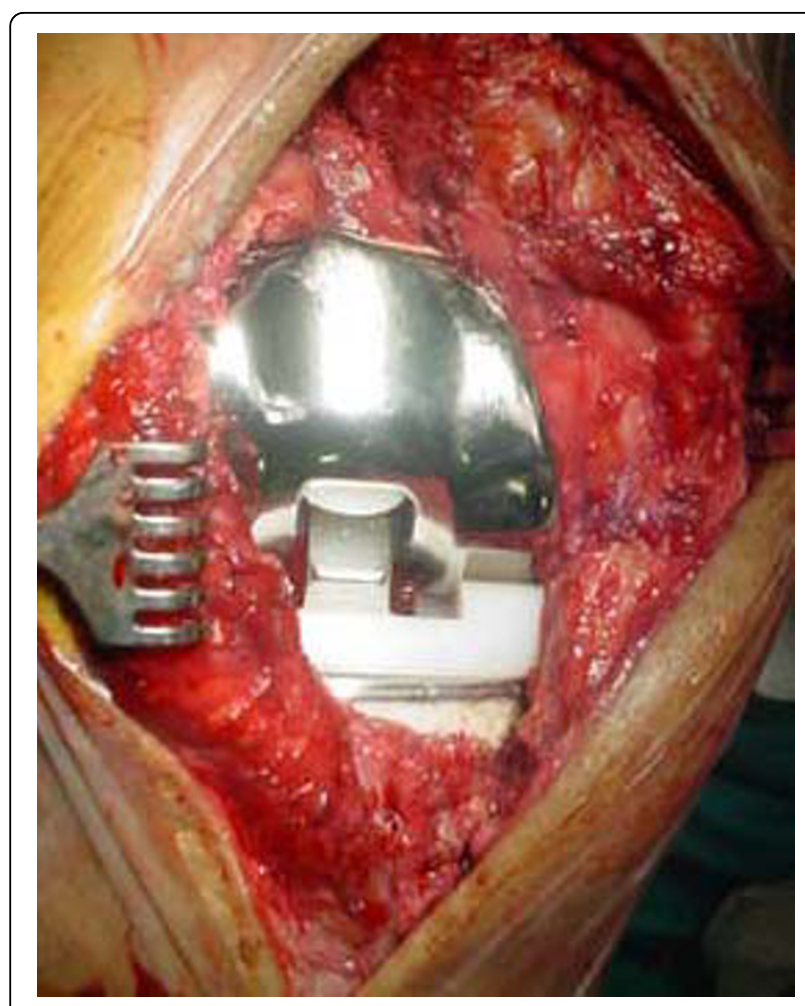

Figure 3 Total knee re-implantation after 3 months. Note the filling of the bone defect in medial aspect of the tibial plateau

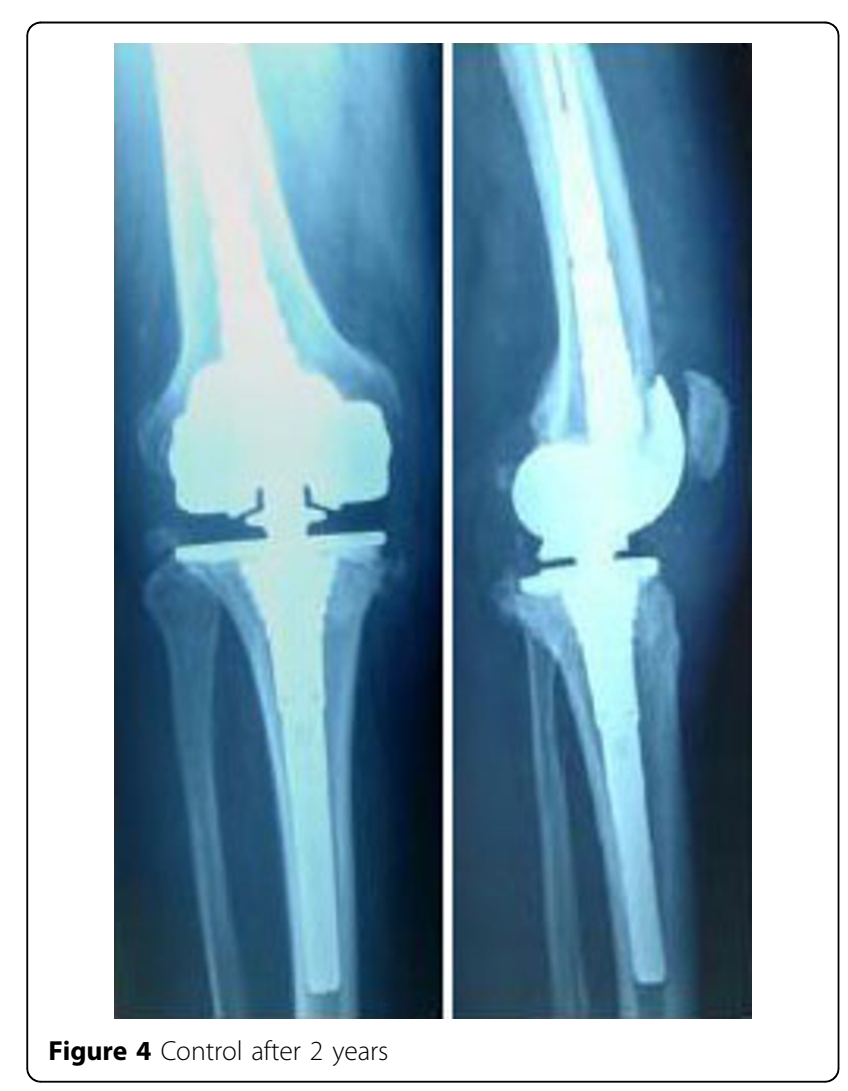

Bone repair and bone mineral density may be significantly retarded and may be corrected by eliminating risk factors, supplementing the diet with calcium, bisphosphonates, and/or vitamin $\mathrm{D}$, and treating with testosterone and/or estrogen when deficient. Sodium fluoride treatment and anabolic steroids may be used as alternatives.

Septic arthritis is a medical emergency, and prompt recognition and rapid and aggressive treatment are critical to ensuring a good prognosis. The treatment of septic arthritis includes appropriate antimicrobial therapy and joint drainage.

Adverse effects of prescribed antibacterials occur more often in the elderly patient than in young adults. The physician can help to minimise the incidence of adverse effects and improve outcomes by being aware of the principles of clinical pharmacology, the characteristics of specific drugs, and the special physical, psychological and social needs of older patients, Figure 1, 2, 3, 4.

Published: 19 May 2010

doi:10.1186/1471-2318-10-S1-L15

Cite this article as: Romanò et al:: Osteomyelitis in elderly patients. BMC Geriatrics 2010 10(Suppl 1):L15.

\section{Submit your next manuscript to BioMed Central and take full advantage of:}

- Convenient online submission

- Thorough peer review

- No space constraints or color figure charges

- Immediate publication on acceptance

- Inclusion in PubMed, CAS, Scopus and Google Scholar

- Research which is freely available for redistribution
C Biomed Central 